\title{
Performance Analysis of Orbital Angular Momentum (OAM) - A 6G Waveform Design
}

\author{
Affan Affan, Shahid Mumtaz, Hafiz M. Asif, and Leila Musavia
}

\begin{abstract}
Next-generation communication systems are required to be efficient in power and spectrum usage. In this direction, novel spatial multiplexing techniques, such as orbital angular momentum (OAM), in contrast to frequency multiplexing, such as orthogonal frequency division multiplexing (OFDM), are regarded to uphold potential advantages. This paper investigates OAM and OFDM along with their emerging variants with index modulation (IM) in MIMO configuration. In this paper, these techniques are implemented for different cell distances mainly for single user cases. The bit error rate (BER) performance analysis is then carried out on a comparative scale. Moreover, Maximum likelihood and least square-based receiver techniques are presented for low complexity detection methods. The strengths and weaknesses of these techniques are analyzed through extensive MATLAB simulations for different Signal-to-Noise Ratios (SNRs).
\end{abstract}

Index Terms-Orbital angular momentum, orthogonal frequency domain multiplexing, index modulation, MIMO channel, maximum likelihood.

\section{INTRODUCTION}

Currently, wireless communication is migrating from $5 \mathrm{G}$ to $6 \mathrm{G}$. This transaction is happening due to the emergence of new use cases and applications, e.g., Sensory Transmission, Teleportation, Extended Reality, Digital Twin, etc. [1], [2]. Academia and industry have already started a quest to find new technologies and solutions to satisfy the explosive data traffic demand of $6 \mathrm{G}$ use cases. The $5 \mathrm{G}$ New Radio (NR) research extensively investigated and proposed various orthogonal resource sharing technologies that exploit the frequency, time, and space characteristics of electromagnetic waves. However, due to bandwidth limitations, it will harden in $6 \mathrm{G}$ if we want to increase capacity or support more users with 5G access techniques such as time-frequency, time, and space. Therefore, 6 G Radio, which we refer to it by enhanced New Radio (eNR), needs to explore another dimension of electromagnetic waves. All 1G-5G wireless communications are built on the planeelectromagnetic (PE) wave. However, the electromagnetic (EM) wave possesses not only linear momentum but also angular momentum, which contains the spin angular momentum (SAM) and orbital angular momentum (OAM) [3]. OAM is one of the physical characteristics of electromagnetic wave propagation. OAM wavefront has helical fronts instead of planar and recently attracted much research attention[4]-[6]. OAM has a significant number of topological charges, i.e.,

Affan A. is with the Electrical and Computer Engineering, University of Louisville, USA (email: affan.affan@louisville.edu).

Shahid M. is with the Instituto de Telecomunicações Campus Universitário de Santiago P-3810-193 AVEIRO - PORTUGAL (email: smumtaz@av.it.pt).

Hafiz M.Asif is with the Electrical and Computer Engineering, Sultan Qaboos University, Muscat, Oman (email: h.asif@squ.edu.om)
OAM-modes [4]. The created beams of different OAM-modes are orthogonal to each other due to successive phase shifts in the OAM models, and they can be multiplexed/demultiplexed together, thus increasing the capacity without relying on the traditional resources such as time and frequency. Therefore, multiple OAM modes transmitted on the same channel simultaneously can be separated and demodulated at receivers. OAM, which has multiple orthogonal topological charges, bridges a new way to increase spectrum efficiency significantly and is expected to be used in $6 \mathrm{G}$ or even more future wireless communications networks.

It is worth mentioning that Orthogonal frequency division multiplexing (OFDM) has been widely used in wireless communication systems due to its advantage in avoiding inter symbol interference and improving SNR. However, OFDM suffers from a high peak to average power ratio (PAPR) and many techniques have been discussed in the literature to combat the high PAPR [7]. In contrast, OAM is inherently power and spectral-efficient [8].

Among many OAM advantages, one of its drawbacks is that it requires a big number of antennas for spatial multiplexing for angle rotation to have spatial orthogonality, which increases its system complexity and RF power consumption. Therefore, to increase its spectral efficiency and reduce power consumption, we combine OAM with the index modulation technique [7][11]. Index modulation (IM) is a digital modulation technique, which uses the ON-OFF keying method to map some of the bits in the transmitted signal to the index of the resources, e.g., the index of the antenna element.

In this paper, OFDM and OAM along with their relative variants based on index modulation (IM), OAM-IM and OFDM-IM, are studied in this research work for a single-user system. The performance of these techniques is compared by calculating the bit error rate (BER) performance under different cell sizes. Our primary studies show the advantages and disadvantages of OAM under various cell sizes and act as a steppingstone toward OAM implementation in the real network.

\section{SYSTEM MODEL}

This section will design the theoretical framework of OFDM, OAM, OFDM-IM, and OAM-IM with two Uniform Circular Arrays (UCAs) in MIMO configuration. System models for each technology are discussed below.

Leila M. is with the School of Computer Science and Electronic Engineering (CSEE), University of Essex, 1NW.4.16, Colchester Campus UK (email: leila.musavian@essex.ac.uk). 


\section{A. Limitations and significance of OFDM}

OFDM is a multi-carrier technique that uses orthogonal carriers with different frequencies [12], [13]. The orthogonality of frequencies helps to avoid inter-symbol interference (ISI) [14]. The block diagram for the OFDM-based communication system is shown in Fig. 1 that explains the major blocks of this technique. The literature about OFDM is well established and more details can be found in [7]-[9].

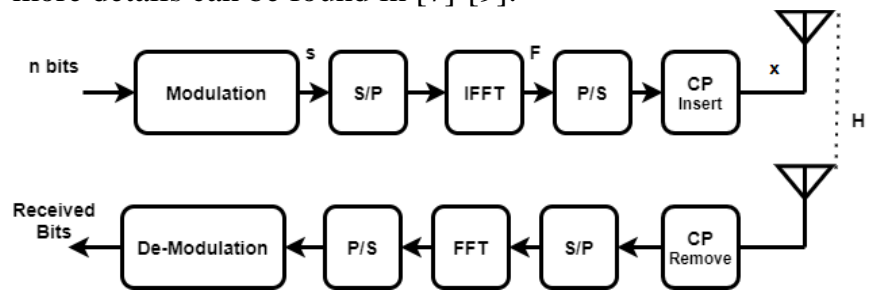

Fig. 1. System block diagram of OFDM.

In OFDM, M-array modulation such as M-PSK or M-QAM is used for improving spectral efficiency. The modulated signal $(\boldsymbol{s})$ is passed through the IFFT block that converts the signal into orthogonal overlapping sinusoids in the time domain. The (1) shows the output of IFFT.

$$
F_{n}=\frac{1}{N} \sum_{t=1}^{N-1} s_{n} \exp \left(j 2 \pi \frac{n t}{N}\right),
$$

A cyclic prefix is added to the OFDM signal. It is helpful to remove inter-symbol interference at the receiver side. The OFDM signal is transmitted through the fading channel as $\boldsymbol{y}=$ $\boldsymbol{H} \boldsymbol{F}+\boldsymbol{n}$, where $\boldsymbol{H} \in \mathbb{R}^{\boldsymbol{N} \times \boldsymbol{N}}$. For $N$ transmitter antennas, the channel coefficient $h_{n g}$ is the channel gain between $n^{\text {th }}$ transmit antenna element of radius $r_{t x}$ and $g^{\text {th }}$ receive antenna element of radius $r_{r x}$ as given in (2) [9].

$$
\begin{gathered}
h_{n g}=\gamma \frac{\lambda}{4 \pi D} \exp (-j k D), \\
D=\sqrt{d^{2}+r_{r x}^{2}+r_{t x}^{2}-2 r_{r x} r_{t x} \cos \varphi_{n g}},
\end{gathered}
$$

where $\varphi_{l g}=\frac{2 \pi(n-g)}{N},\left(n\right.$ is the $n^{\text {th }}$ transmit antenna). The cyclic prefix is removed on the receiver side, and the signal is passed through the FFT block. The FFT block's output is processed through the least square error technique to recover the information bits. OFDM has been a pivotal technique in communication systems, however, due to high data traffic OFDM based systems are unable to support a higher number of users without exhausting frequency bandwidth. To increase the spectral efficiency, OAM exploits the angular momentum of the EM waves which can accommodate more users within a single frequency band due to inherent orthogonality in OAM modes, unlike OFDM which depends on orthogonal frequencies. OAM with MIMO configuration also increases the spatial diversity of communication systems. OAM-based systems have comparatively simple transmit/receive structures which give OAM an advantage over OFDM.

\section{B. Orbital Angular Momentum (OAM)}

The OAM is a type of spatial multiplexing that exploits orbital properties of electromagnetic (EM) waves, which uses both radial and azimuthal degrees of freedom multiplexing and demultiplexing [3]. In OAM, multiple copies of the same signal are fed to all antennas but with a phase shift. After a complete cycle, the phase will be increased by an integer multiple of $2 \pi$. The OAM wave is generated by a phase rotation factor $\exp (-j k \rho)$, where $j$ is the complex number, $k$ is the order of OAM-mode, and $\rho$ is the azimuthal angle such as $2 \pi$ [15]. Different OAM-modes are orthogonal with each other due to phase shifts. With UCAs, it is easy to construct OAM mode by using discrete Fourier transform (DFT) for multiplexing [16]. The OAM wave can be demultiplexed by using inverse discrete Fourier transform (IDFT). Fig. 2 shows the block diagram of the communication system using OAM. The system consists of the ' $\beta$ ' number of bits and $N$ antenna elements. The bits are modulated by using PSK or QAM with modulation order $M$ and $s$ is the modulated signal. To create an OAM beam, the modulated signal is multiplied with the DFT matrix as:

$$
\hat{x}_{n}=\frac{1}{\sqrt{N}} s_{n} \exp \left(-j 2 \pi \frac{n}{N}\right),
$$

where $n \in\{0,1 \cdots N-1\}$, shows the index of element of the antenna, which is being fed with the information signal. For the transmission, Eqns (5) show the allocation of power $p$ to the signal $\hat{x}$.

$$
\begin{gathered}
x_{n}=\hat{x}_{\mathrm{n}} p_{n}, \\
p_{n}=\sqrt{\frac{P_{t} / \delta_{n}}{\frac{1}{\delta_{1}}+\frac{1}{\delta_{2}}+\cdots \frac{1}{\delta_{N-1}}}}
\end{gathered}
$$

where $\delta_{i}$ are the eigenvalues of channel $\boldsymbol{H}$ and $P_{t}$ is total transmission power. The signal $\boldsymbol{x}$ is passed through the fading channel $\boldsymbol{H}$, using the channel defined in(2). Equations (4) to (6) can be written in vector form as follows:

$$
\begin{gathered}
\widehat{x}=W s, \\
x=\widehat{x} P, \\
y=H x+n,
\end{gathered}
$$

where $\boldsymbol{s} \in \mathbb{C}^{N \times 1}$ is the modulated signal, $\boldsymbol{W} \in \mathbb{C}^{N \times N}$ is the discrete Fourier transform matrix, $\boldsymbol{P}=\operatorname{diag}\left(p_{0} p_{1} \cdots p_{N}\right)$ is the power matrix allocated to each symbol, $\boldsymbol{n} \in \mathbb{R}^{N \times 1}$ is the white Gaussian noise, $\boldsymbol{x}$ is the signal after the DFT, $\widehat{\boldsymbol{x}}$ is the signal with power allocation and $y \in \mathbb{C}^{N \times \mathbf{1}}$ is the signal to be transmitted. Equation (8) shows the demultiplexing of received signal $\boldsymbol{y}$ using inverse Fourier transform.

$$
y_{r}=\frac{1}{\sqrt{N}} \sum_{t=1}^{N-1} y_{t} \exp \left(j 2 \pi \frac{p_{r}}{N}\right),
$$

where $y_{t}$ is the $t^{t h}$ element of transmitted signal passed through fading channel and $y_{r}$ is the $r^{\text {th }}$ element of the received signal, $r=0,1, \cdots, N-1$. The information bits are recovered using the least square error technique.

\section{OFDM with Index Modulation (IM)}

The OFDM-based communication system is discussed in II.A. IM with OFDM introduces the mode activation fashion in the 
frequency domain by selecting a certain number of OFDM subcarriers at a time and mapping the extra information in the indexes of these activated subcarriers [17]. It helps to reduce the bandwidth usage and power consumption like spatial multiplexing,

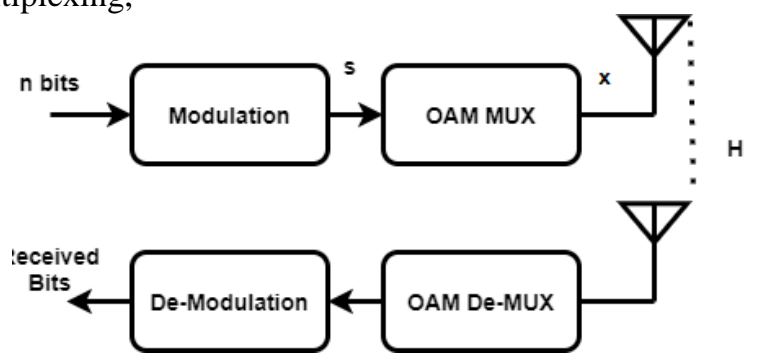

Fig. 2. System block diagram of OAM.

however, in OFDM-IM, the number of antennas does not limit the implementation of IM. The block diagram of OFDM-IM based communication system is shown in Fig. 3 .

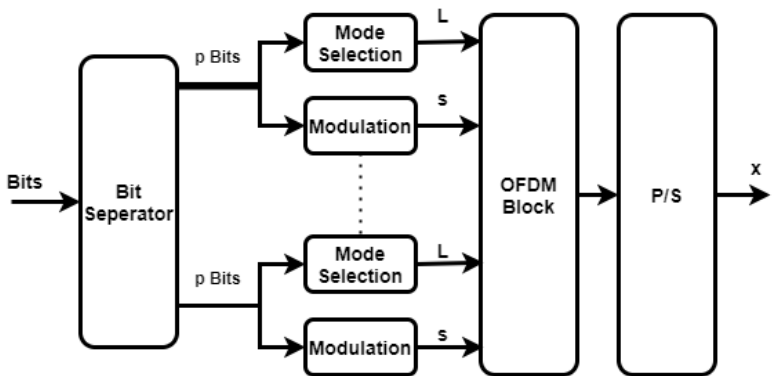

Fig. 3. System block diagram of OFDM-IM Transmitter

The $\beta$ bits are separated into $G$ subblocks. Each subblock contains $p$ bits, $p=N / G$, where $N$ is the number of subcarriers. These $p$ bits are subdivided into $p_{1}$ bits required for modulation and $p_{2}$ used for mode selection. Out of these $N$ subcarriers, only $N_{a}$ activated subcarriers are used for data transmission. The information about these activated subcarriers is stored in $\mathcal{M}_{i}=\left\{i_{1}, l_{2}, \cdots l_{N_{a}}\right\}$, where the $i^{\text {th }}$ combination of activated modes based on mode selection bits. Equation (9) shows the spectral efficiency of OFDM-IM.

$$
\beta=G N_{a} \log _{2} M+\left\lfloor\log _{2}\left(\begin{array}{c}
N \\
N_{a}
\end{array}\right)\right\rfloor G .
$$

The modulated signal in each subblock is mapped to $N_{a}$ subcarriers and other subcarriers are forced to be zero. The output of each subblock is converted to the serial mode and passed through fading channel $H$. The transmitted signal $\boldsymbol{x}$ can be expressed as follows:

$$
x=[\widehat{x}(1), \widehat{x}(2) \cdots \widehat{x}(N)],
$$

where elements of $\boldsymbol{x}$ represent each of G subblocks which can be written as follows.

$$
\widehat{x}=\left[s_{1}, s_{2}, \cdots s_{N_{a}}\right] \text {. }
$$

The subblock signal $\widehat{\boldsymbol{x}}$ contains non-zero constellation symbols and zero values. The transmitted bits are recovered by using the maximum likelihood detector. First, indexes of subcarriers carrying information bits with respect to activation mode combination should be detected [9]. The detection of subcarriers with information bits can be identified using (12) and (13).

$$
\begin{gathered}
\xi_{u}^{i}=\frac{\left|y_{r_{u}}\right|^{2}}{\sigma_{n}^{2}}+\ln \left(\sum_{d \in S e j \theta i} \exp \left(-\frac{1}{\sigma_{n}^{2}}\left|y_{r_{u}}-g_{u} \mathrm{~d}\right|^{2}\right)\right), \\
\xi^{i}=\sum_{u \in \mathcal{M}_{i}} \xi_{u}^{\left|\frac{k N_{a}}{N}\right|},
\end{gathered}
$$

where $y_{r}$ is the received signal after demultiplexing OAM. The possible $i^{\text {th }}$ combination can be detected by calculating the LLR sum of each mode combination.

$$
\hat{\imath}=\arg \max _{i} \xi^{i}
$$

The symbols can be detected as follows:

$$
\hat{d}_{u}=\operatorname{argmin}\left|y_{r_{u}}-g_{u} d\right|, u \in \mathcal{M}_{\hat{\imath}}
$$

\section{OAM with Index Modulation (IM)}

In subsection II.B, the OAM based communication system is discussed. To increase the spectral efficiency and reduce the power requirement, index modulation is used in addition to OAM. The active OAM modes are used to transmit additional bits. The total number of activated modes is equal to the number of transmit antennas donated as $N$. It is assumed that same number of transmit, and receiver antennas are used. The number of activated modes is $N_{a}$, where $N_{a} \in[1,2, \cdots N]$ and $u$ is desired activated mode [9]. The activated mode equal to $N$ that all OAM modes are used for transmission is known as OAM based mode division multiplexing (MDM). The spectral efficiency of the OAM-IM can be calculated as follows:

$$
\beta=\mathrm{N}_{\mathrm{a}} \log _{2} M+\left\lfloor\log _{2}\left(\begin{array}{c}
N \\
N_{a}
\end{array}\right)\right\rfloor,
$$

where $M$ is the modulation order. The block diagram of OAMIM based communication system is shown in Fig. 4.

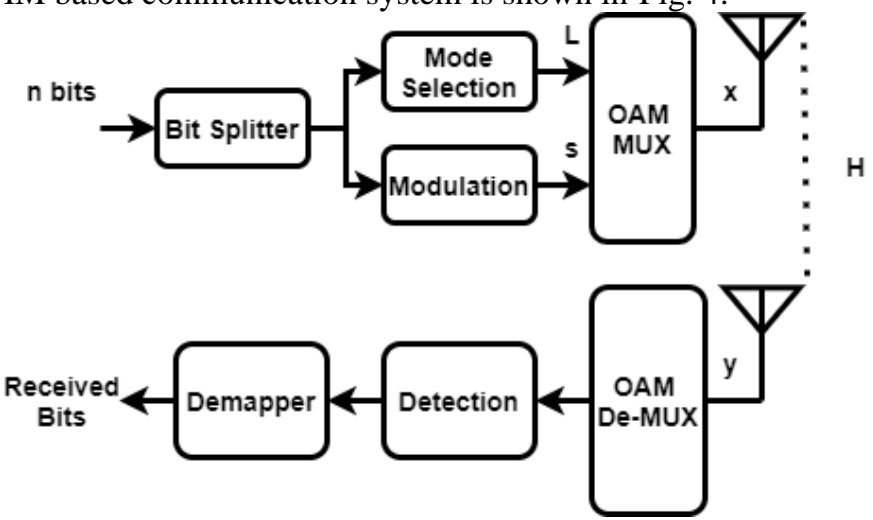

Fig. 4. System block diagram of OAM-IM

The $\mathrm{N}_{\mathrm{a}} \log _{2} M$ bits are used for modulation and $\left[\log _{2}\left(\begin{array}{c}N \\ N_{a}\end{array}\right)\right]$

$$
x_{n}^{u}=\frac{1}{\sqrt{N}} p_{u} s_{u} \exp \left(-j 2 \pi \frac{n u}{N}\right),
$$

where $p_{u}$ is the power allocated to the mode $u, s_{u}$ is the $u^{\text {th }}$ element of the modulated signal. The vector $\boldsymbol{s}$ contains complex constellation symbols and some zeros, the indexes of non-zero elements is stored in $\boldsymbol{M}$. With OAM-IM, the antenna element $n$ is fed by the linear superposition of the signal of 


$$
\begin{gathered}
x_{n}=\sum_{u=\mathcal{M}_{i}} x_{n}^{u}=\frac{1}{\sqrt{N}} p_{u} s_{u} \exp \left(-j 2 \pi \frac{n u}{N}\right), \\
p_{n}=\sqrt{\frac{P_{t} / \delta_{n}}{\frac{1}{\delta_{1}}+\frac{1}{\delta_{2}}+\cdots \frac{1}{\delta_{N-1}}}}
\end{gathered}
$$

Equation (17) and (18) can be written in vector form as follows:

$$
\boldsymbol{x}=W P s,
$$

where $\boldsymbol{W} \in \mathbb{C}^{N \times 1}$ is discrete Fourier transform matrix and $\boldsymbol{P}$ is the power allocation matrix. The vector $s \in \mathbb{C}^{N \times \mathbf{1}}$ contains complex constellation symbols $d \in S e^{j \theta i}$ and some zeros, the indexes of non-zero elements is stored in $\mathcal{M}_{i}$. Vector $\boldsymbol{s}$ is represented by:

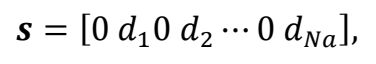

The signal $\boldsymbol{x}$ is passed through the fading channel $H$ defined in (2). On the receiver side, the received signal is de-multiplexed by using inverse discrete Fourier transform matrix as follows [9], [11]:

$$
\begin{gathered}
y=H x+n, \\
W^{H} y=W^{H} H x+W^{H} n, \\
y_{r}=W^{H} H W P s+W^{H} n, \\
y_{r}=\Lambda P s+W^{H} n, \\
y_{r}=G s+W^{H} n,
\end{gathered}
$$

where $\boldsymbol{y}_{\boldsymbol{r}}$ is the received signal and $\boldsymbol{\Lambda}$ is a diagonal matrix defined as $\boldsymbol{\Lambda}=\operatorname{diag}(\operatorname{eig}(\boldsymbol{H}))$. The maximum likelihood detector is used based on LLR calculations to identify the indexes of activated modes and recover the transmitted bits as given in (12)-(15).

\section{Simulation Results}

For comparison purposes, the techniques mentioned above are implemented in MATLAB for a single user with different cell distances. For the sake of consistency, the order of modulation, the number of the transmit and the receive antennas, and the number of activated modes are kept the same in each of these simulated techniques. The simulation parameters are given in The elements of $\boldsymbol{M}$ show the activated mode in space and frequency domain. The bit error rate (BER) of OAM and OFDM for different cell distances are shown in Fig. 5. It is important to mention that all four techniques are compared using a similar number of activated . The mode combination matrix for $4 \times 4$ antenna configuration with two activated modes is given in (23).

TABLE I

Simulation Parameters.

\begin{tabular}{lc}
\hline \hline \multicolumn{1}{c}{ Parameter } & Value \\
\hline Operating frequency & $10 \mathrm{GHz}$ \\
Wavelength & $0.03 \mathrm{~m}$ \\
Antenna radius & $0.6 \mathrm{~m}$ \\
Antenna configuration & $4 \times 4$ \\
Average transmission Power & $1 \mathrm{~W}$
\end{tabular}

$$
\boldsymbol{M}=\left[\begin{array}{ll}
0 & 1 \\
2 & 3 \\
0 & 2 \\
1 & 3
\end{array}\right]
$$

The elements of $\boldsymbol{M}$ show the activated mode in space and frequency domain. The bit error rate (BER) of OAM and OFDM for different cell distances are shown in Fig. 5. It is important to mention that all four techniques are compared using a similar number of activated modes.

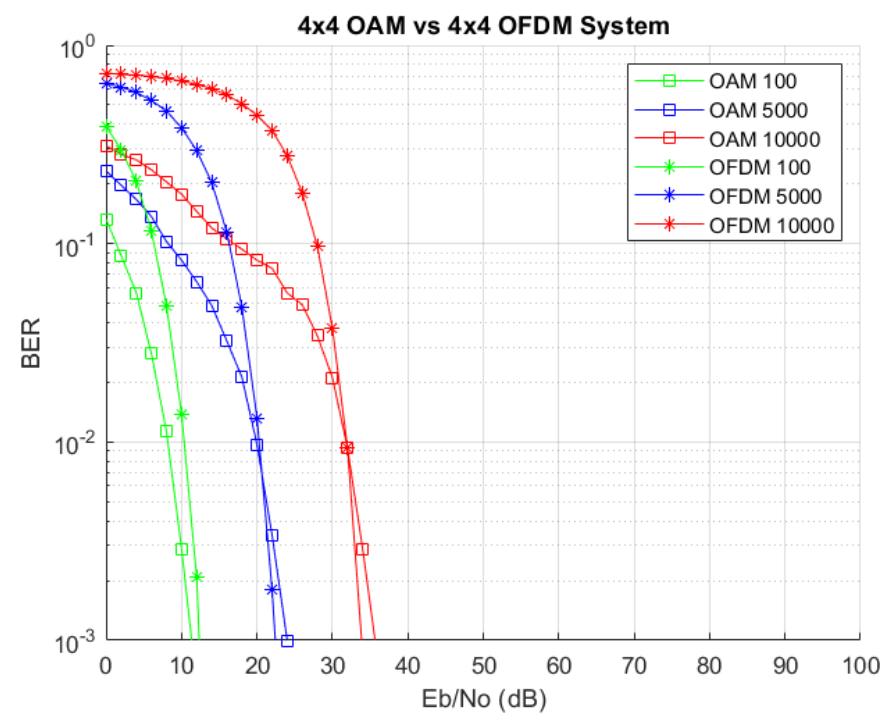

Fig. 5. BER of OFDM versus BER of OAM for different cell distances.

Comparing the BER of OAM and OFDM shows that the distance impacts the BER of various techniques significantly. Fig. 5 shows that the OAM performed better for small SNR values for all ranges of distance. However, OFDM and OAM performed approximately equal for higher values of SNR for all values of cell distances. It is important to discuss that LLR in OAM performed better as compared to the least square method in OFDM, which highlights the significance of simpler transmit/receiver design in OAM based systems. Moreover, the use of different rotation angles $\left(\theta_{2}\right)$ for different OAM modes in M-PSK can also increase the performance of the OAM system. For the sake of simplicity, a rotation angle equal to zero is considered in this simulation.

The BER of OAM-IM and OFDM-IM is shown in Fig. 6. The comparison of BER of OAM-IM and OFDM-IM shows that both techniques performed approximately equal for short-range distances such as $100 \mathrm{~m}$ and $5 \mathrm{Km}$. However, for long-distance, such as $10 \mathrm{Km}$, the BER graph shows considerable difference in both techniques. For long-distance scenarios, OAM-IM performed better for low SNR values, however, OFDM-IM performed slightly better for high SNR values in this scenario. This shows that OAM based techniques have a trade-off between spectral efficiency and BER performance. This tradeoff can be balanced by using a higher number of OAM modes which will increase the complexity of the system design. 


\section{CONCLUSIONS}

We have investigated the orthogonal frequency division multiplexing (OFDM) and orbital angular momentum multiplexing (OAM) along with their relative variants based on index modulation (IM), OAM-IM and OFDM-IM, in this research work for a single-user system. The simulation results have shown that OFDM based communication system performs better for high signal-to-noise ratio (SNR) values while OAM based systems better for low SNR values. The addition of IM with OAM and OFDM increases the system's efficiency and reduces the difference for short-range communication. Based on the results, it can be concluded that OAM has significantly more spectral efficiency than OFDM and IM improves the spectral efficiency as well as BER. Based on this work, the future work aims to design communication systems for multiusers using these techniques.

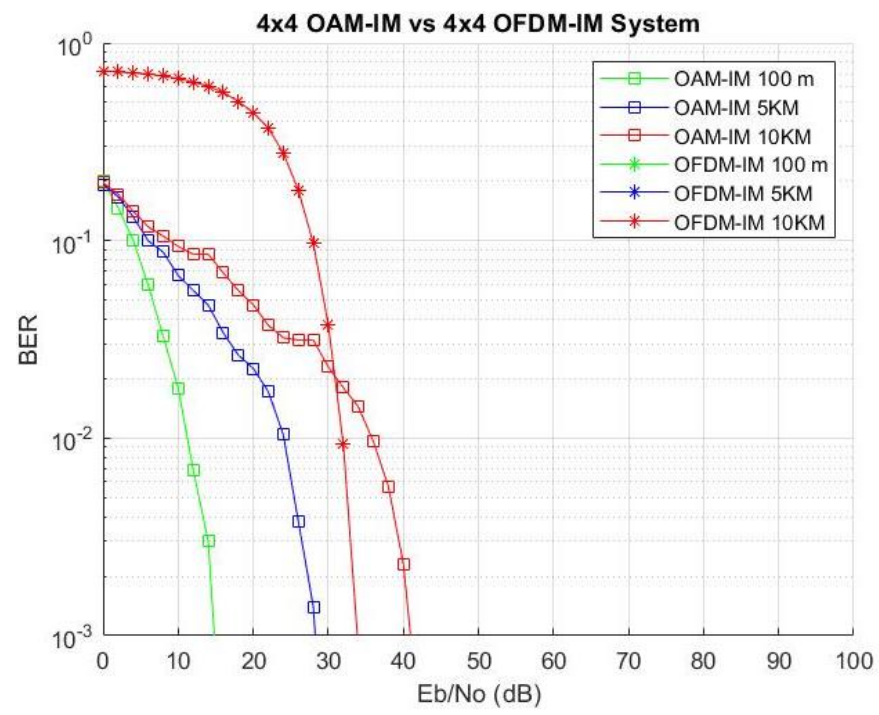

Fig. 6. BER of OFDM-IM versus BER of OAM-IM for different cell distances.

\section{REFERENCES}

[1] P. Yang, Y. Xiao, M. Xiao, and S. Li, "6G Wireless Communications: Vision and Potential Techniques," IEEE Netw, vol. 33, no. 4, pp. 70-75, Jul. 2019.

[2] M. Z. Chowdhury, M. Shahjalal, S. Ahmed, and Y. M. Jang, "6G wireless communication systems: Applications, requirements, technologies, challenges, and research directions," arXiv. arXiv, Sep. 25, 2019.

[3] W. Cheng, W. Zhang, H. Jing, S. Gao, and H. Zhang, "Orbital angular momentum for wireless communications," IEEE Wirel Commun, vol. 26, no. 1, pp. 100-107, Feb. 2019.

[4] B. Thidé et al., "Utilization of photon orbital angular momentum in the low-frequency radio domain," Phys Rev Lett, vol. 99, no. 8, May 2007.

[5] X. Xiong, S. Zheng, Z. Zhu, X. Yu, X. Jin, and X. Zhang, "Performance Analysis of Plane Spiral OAM Mode-Group Based MIMO System," IEEE Commun Lett, vol. 24, no. 7, pp. 1414-1418, Jul. 2020.
Beam Selection for Indoor Millimeter Wave MUMIMO Systems," IEEE Commun Lett, vol. 25, no. 5, pp. 1702-1706, May 2021.

[7] A. Sharma and A. Kr. Singh, "Orthogonal Frequency Division Multiplexing and its applications," Int $J$ Comput Trends Technol, vol. 38, no. 1, pp. 21-23, 2016.

[8] R. Chen, H. Zhou, W. X. Long, and M. Moretti, "Spectral and energy efficiency of line-of-sight OAMMIMO communication systems," China Commun, vol. 17, no. 9, pp. 119-127, 2020.

[9] E. Basar, "Orbital angular momentum with index modulation," IEEE Trans Wirel Commun, vol. 17, no. 3, pp. 2029-2037, Mar. 2018.

[10] Q. Li, M. Wen, B. Clerckx, S. Mumtaz, A. AlDulaimi, and R. Q. Hu, "Subcarrier Index modulation for future wireless networks: Principles, applications, and challenges," IEEE Wirel Commun, vol. 27, no. 3, pp. 118-125, Jun. 2020.

[11] L. Liang, W. Cheng, W. Zhang, and H. Zhang, "Mode hopping with OAM-based index modulation," Dec. 2019.

[12] A. Affan, U. Khan, H. M. Asif, and K. Raahemifar, "Multiuser visible light communication system using hybrid OFDM-PWM," 2020.

[13] G. L. Stüber, J. R. Barry, S. W. Mclaughlin, Y. E. Li, M. A. Ingram, and T. G. Pratt, "Broadband MIMOOFDM wireless communications," in Proceedings of the IEEE, 2004, vol. 92, no. 2, pp. 271-293.

[14] M. Ahsan and H. M. Asif, "ESIM-OFDM-based transceiver design of a visible light communication system," Int J Commun Syst, vol. 30, no. 8, p. e3175, May 2017.

[15] K. A. Opare, Y. Kuang, and J. J. Kponyo, "Mode combination in an ideal wireless OAM-MIMO multiplexing system," IEEE Wirel Commun Lett, vol. 4, no. 4, pp. 449-452, Aug. 2015.

[16] O. Edfors and A. J. Johansson, "Is orbital angular momentum (OAM) based radio communication an unexploited area?," IEEE Trans Antennas Propag, vol. 60, no. 2 PART 2, pp. 1126-1131, Feb. 2012.

[17] E. Basar, Ü. Aygölü, and E. Panayirci, "OFDM için yeni bir teknik: OFDM-indis modülasyonu,” 2013.

[18] E. Basar, M. Wen, R. Mesleh, M. Di Renzo, Y. Xiao, and H. Haas, "Index Modulation Techniques for NextGeneration Wireless Networks," IEEE Access, vol. 5, pp. 16693-16746, Aug. 2017. 